\title{
Laser scribing of fluorinated polyimide films to generate microporous structures for high- performance micro-supercapacitor electrodes
}

Minsu Kim, ${ }^{\dagger, \#}$ Min Guk Gu, ${ }^{\ddagger}, \#$ Heeyoung Jeong, ${ }^{\dagger, \S}$ Eunseok Song, ${ }^{\ddagger}$ Jun Woo Jeon, ${ }^{\dagger}$ KangMoo Huh, ${ }^{\S}$ Pilgyu Kang, ${ }^{*}$, Sung-Kon Kim, ${ }^{*},+$ Byoung Gak Kim ${ }^{*},, \perp$

$\dagger$ Advanced Materials Division, Korea Research Institute of Chemical Technology, 141 Gajeongro, Yuseong-gu, Daejeon 34114, Republic of Korea

$\$$ School of Chemical Engineering, Jeonbuk National University, 567 Baekje-daero, Deokjingu, Jeonju-si, Jeolabuk-do, 54896, Republic of Korea

\$Department of Polymer Science and Engineering, Chungnam National University, 99 Daehakro, Yuseong-gu, Daejeon, Republic of Korea

"Department of Mechanical Engineering, George Mason University, Fairfax, VA 22030, USA

${ }^{\perp}$ Department of Chemical Convergence Materials and Process, University of Science and Technology, 217 Gajeongro, Yuseong-gu, Daejeon 34114, Republic of Korea

\#These authors contributed equally.

\section{Corresponding Authors:}

"E-mail: pkang7@,gmu.edu

"E-mail: yunho@krict.re.kr

*E-mail: bgkim@krict.re.kr

\section{List of contents:}

1. Supporting Experimental Methods

2. Supporting Figures

3. Supporting Tables

4. Supporting References 


\section{Supporting Experimental Methods}

\section{Materials}

4,4'-(Hexafluoroisopropylidene)diphthalic anhydride (6FDA) and 2,2'-bis(trifluoromethyl)-[1,1'biphenyl]-4,4'-diamine (TFB) were purchased from Changzhou Sunlight Pharmaceutical Co., Ltd. 6FDA and TFB were purified by vacuum sublimation and recrystallization from methanol, respectively, and were dried under vacuum at $80^{\circ} \mathrm{C}$ for $24 \mathrm{~h}$ prior to the synthesis of poly(amic) acids (PAA) solution. Anhydrous N,N-Dimethylacetamide (DMAc) was purchased from Sigma-Aldrich and used as received without further purification.

\section{Synthesis of poly(amic) acids (PAA) and fluorinated aromatic polyimides films (fPI)}

PAA was prepared by solution polymerization in a 1L 3-neck flat bottom type reaction flask equipped with a mechanical stirrer for mixing under nitrogen atmosphere. TFB (32.024 g, $0.1 \mathrm{~mol})$ was added to $326 \mathrm{ml}$ of DMAc and stirred at room temperature until the solution was homogeneous. Then, the stoichiometric equivalent amount of 6FDA $(44.424 \mathrm{~g}, 0.1 \mathrm{~mol})$ was added to the solution in an ice bath and stirred. After mixing 6FDA into the solution for $1 \mathrm{~h}$, the mixture was vigorously stirred for $8 \mathrm{~h}$ at room temperature. After the reaction, viscous PAA solution with $20 \mathrm{wt} \%$ solid content was obtained. To facilitate casting the fPI films, $12 \mathrm{wt} \%$ PAA solution was prepared by adding the appropriate amount of DMAc to $20 \mathrm{wt} \%$ PAA solution. Before proceeding the thermal imidization process, $12 \mathrm{wt} \%$ PAA solution (12.90 g) was poured into a glass petri dish and heated under vacuum to slowly evaporate the solvent and stabilize the films. After the stabilization step, the film was imidized under nitrogen atmosphere using a heat treatment process that further heated to different temperatures (Figure S1). The conditions in stabilization step and imidization step of the films used in this study are summarized in the Table S1. The chemical structure of fPI was confirmed by Fourier-transform infrared (FT-IR), ${ }^{1} \mathrm{H}$-nuclear magnetic resonance ( $\left.{ }^{1} \mathrm{H}-\mathrm{NMR}\right)$ spectroscopy. In the FT-IR spectra, the C-C stretching (1489 $\mathrm{cm}^{-1}$ ) peak by the benzene ring was confirmed. The characteristic peaks of asymmetric $\mathrm{C}=\mathrm{O}$ stretching $\left(1785 \mathrm{~cm}^{-1}\right)$, symmetric $\mathrm{C}=\mathrm{O}$ stretching vibration $\left(1725 \mathrm{~cm}^{-1}\right)$ and $\mathrm{C}=\mathrm{O}$ bending $\left(718 \mathrm{~cm}^{-1}\right)$ were found and the imide ring structure was confirmed. The stretching $\left(1362 \mathrm{~cm}^{-1}\right)$ peak of the C-N-C bond in the imide ring structure and the characteristic $1120-1350 \mathrm{~cm}-1$ peaks of the functional group $-\mathrm{CF}_{3}$ in $\mathrm{fPI}$ 
were shown. The $-\mathrm{NH}$ stretching $\left(1540 \mathrm{~cm}^{-1}\right)$ and $\mathrm{C}=\mathrm{O}$ stretching $\left(1650 \mathrm{~cm}^{-1}\right)$ peaks of amide group in PAA were hardly seen (Figure S2). In the ${ }^{1} \mathrm{H}-\mathrm{NMR}$ spectra, the hydrogen atoms in imide ring structure were observed between $7 \mathrm{ppm}$ and $10 \mathrm{ppm}$. The $-\mathrm{COOH}$ proton peak from $12 \mathrm{ppm}$ to $14 \mathrm{ppm}$ and CONH proton peak from $10.5 \mathrm{ppm}$ to $11.5 \mathrm{ppm}$ were negligible (Figure S3). We further characterized thermal and optical properties of fPI, using thermogravimetric Analysis (TGA) and Ultraviolet-visible (UV-vis) spectroscopy, respectively. The TGA curve of fPI film shows that the decomposition temperature of fPI film at $5 \mathrm{wt} \%$ loss was evaluated to be $521.3^{\circ} \mathrm{C}$ (Figure $\mathbf{S 4}$ ). The UV-vis spectra of fPI film shows the light transmittances of fPI at visible range $(400 \sim 740 \mathrm{~nm})$ were evaluated to be 84.5 90.4\% (Figure S5). Based on the chemical, thermal, and optical characterization of the fPI film, we confirmed the PAA was imidized well to fPI.

Characterization of poly (amic) acids (PAA) and fluorinated aromatic polyimides films (fPI)

Proton nuclear magnetic resonance (1H-NMR) spectrum of PAA were measured at $500 \mathrm{MHz}$ on a Bruker AVANCE NEO, using chloroform- $d\left(\mathrm{CDCl}_{3}\right)$ as solvent. An attenuated total reflection-Fourier transform infrared spectroscope (ATR-FTIR) (Nicolet 5700, Thermo Fisher Scientific) was used to analyze the chemical structure and chemical bonds of the fPI. Thermogravimetric anaylsis (TGA) (TGA Q500, TA Instrument) was used to analyze the thermal properties of the fPI under nitrogen flow at heating rate of $10{ }^{\circ} \mathrm{C} / \mathrm{min}$. UV-vis spectrophotometer (Cary 5000, Varian) was used to characterize optical properties of fPI. 


\section{Supporting Figures}<smiles></smiles>

6FDA

TFB

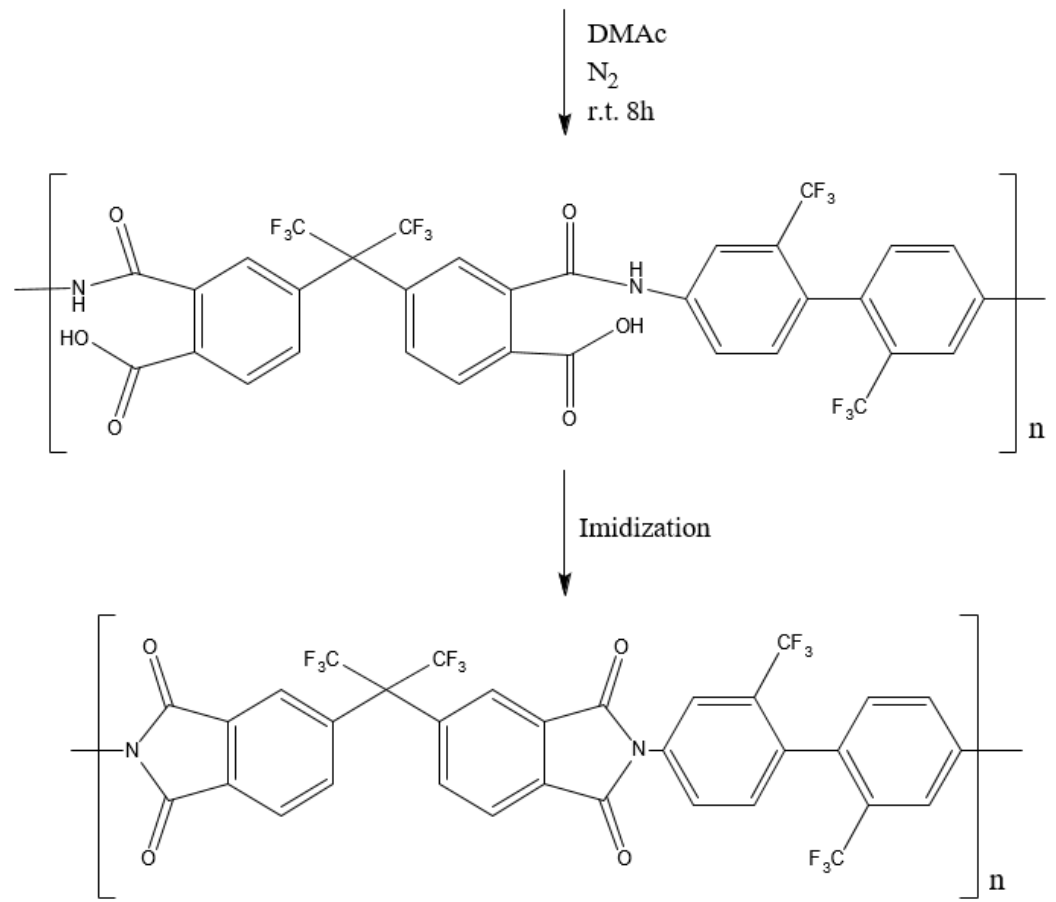

Figure S1 Synthetic route to poly(amic) acid (PAA) and fluorinated aromatic polyimide (fPI). 


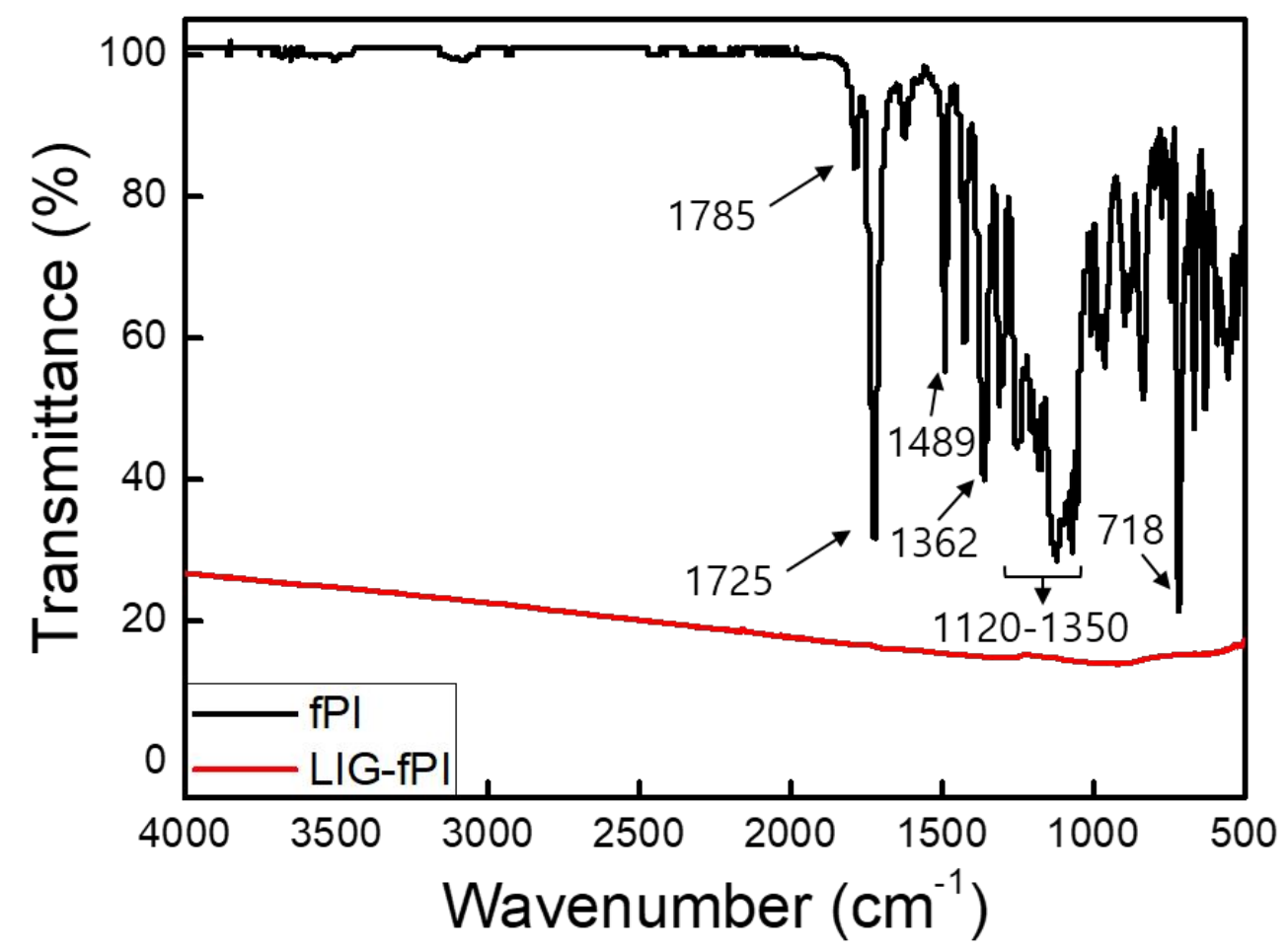

Figure S2 Fourier-transform infrared (FT-IR) spectra of fPI and LIG-fPI films. 


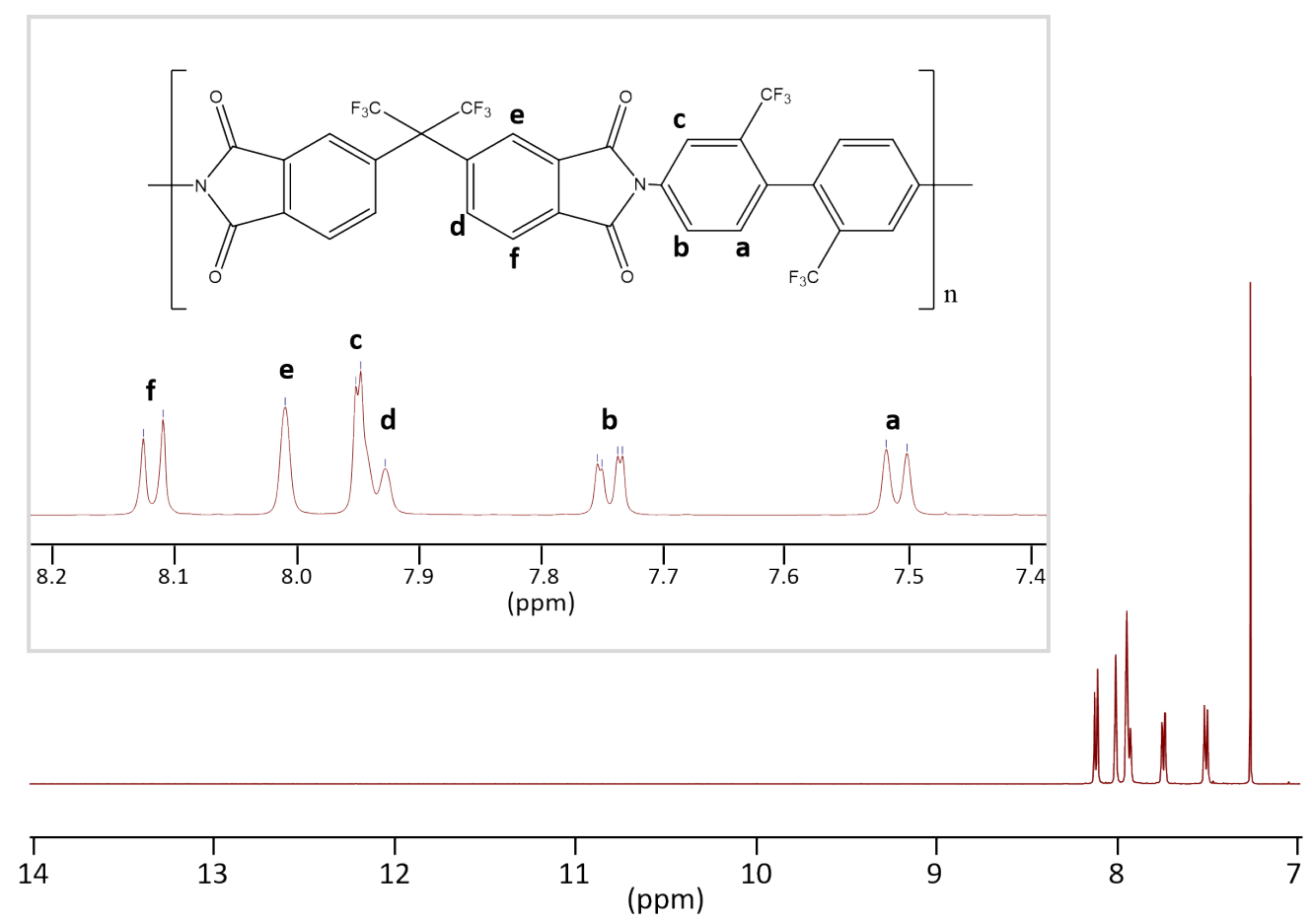

Figure $\mathbf{S 3}{ }^{1} \mathrm{H}-\mathrm{Nuclear}$ magnetic resonance $\left({ }^{1} \mathrm{H}-\mathrm{NMR}\right)\left(\mathrm{CDCl}_{3}, 500 \mathrm{MHz}\right)$ spectra of fPI. 


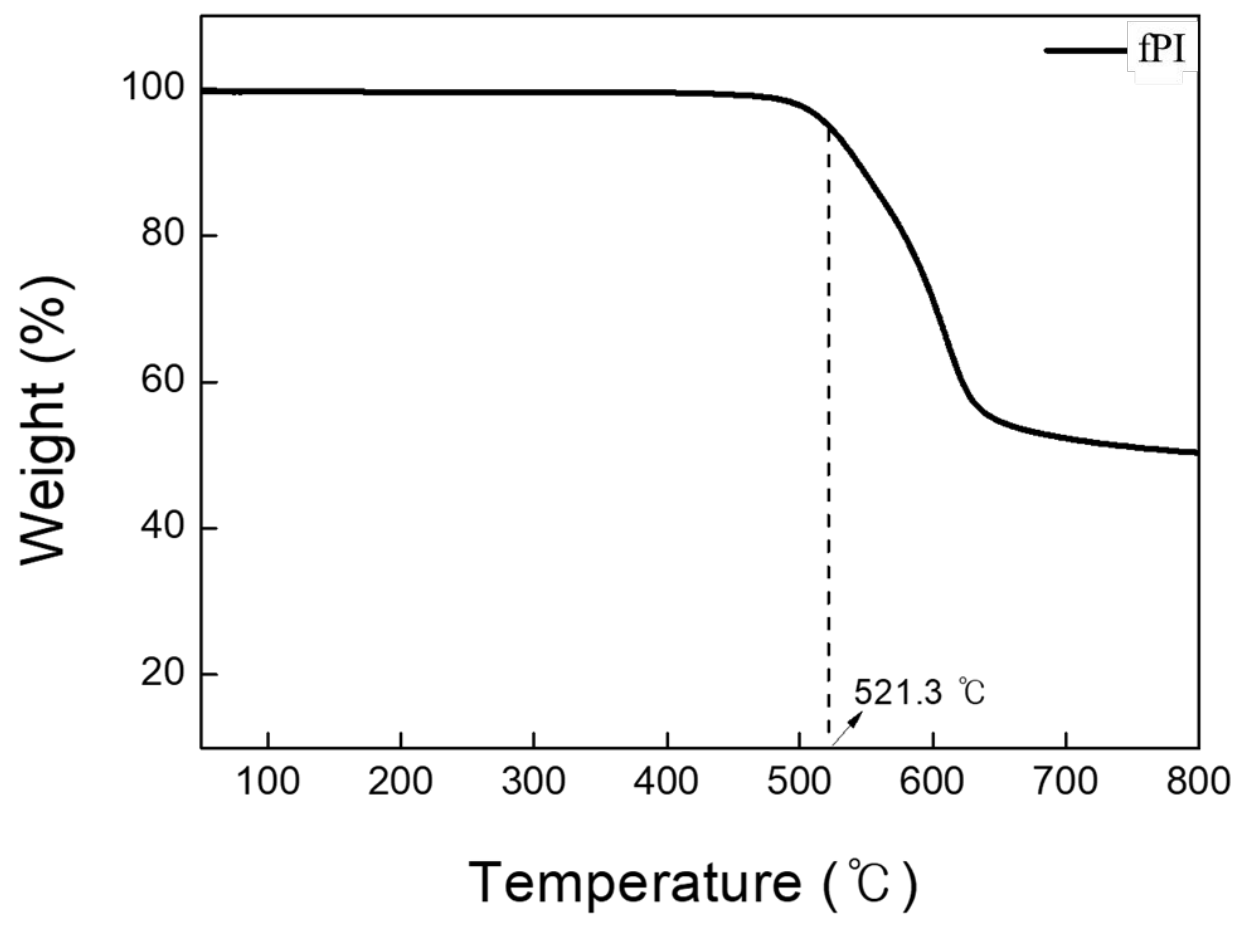

Figure S4 Thermogravimetric Analysis (TGA) curve of fPI film. 


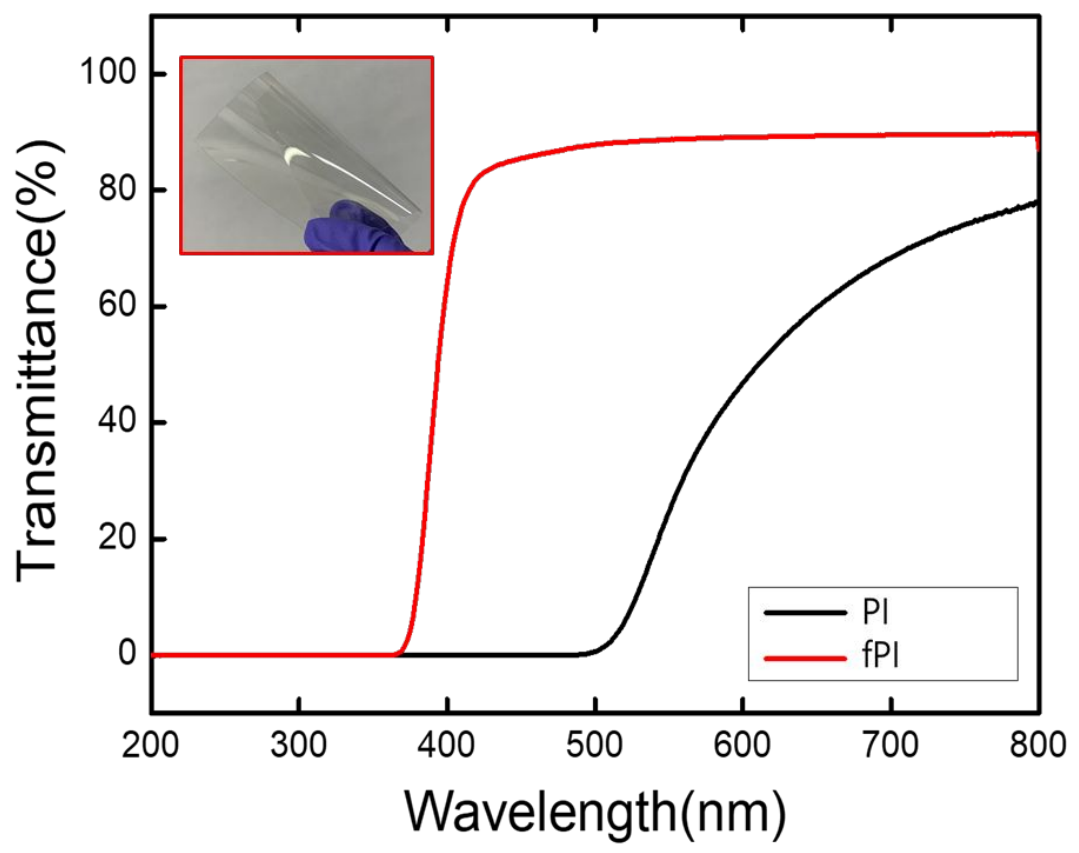

Figure S5 Ultraviolet-visible (UV-vis) spectrum of fPI and PI films. 
a

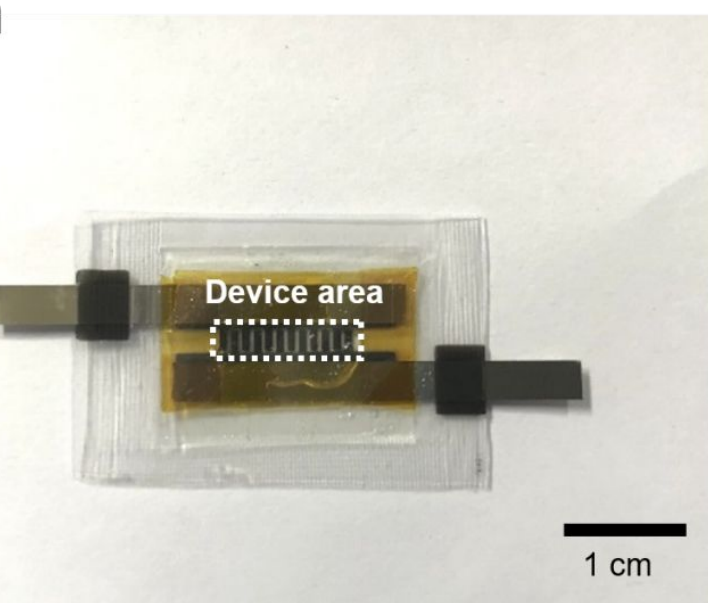

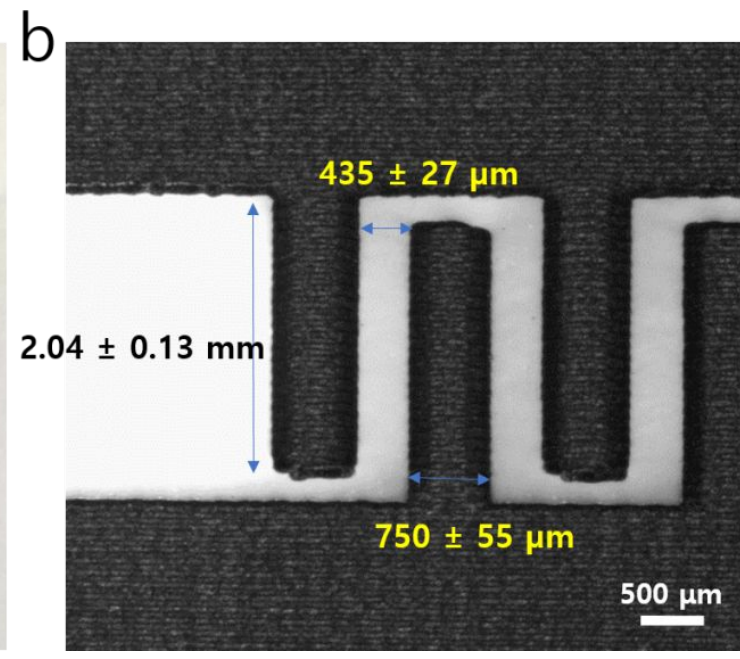

Figure S6 (a) Photograph of LIG-fPI-MSC pouch cell employing o-GPE. Dashed line in figure a denotes the area of device. (b) Top SEM image of the micro-electrodes. 


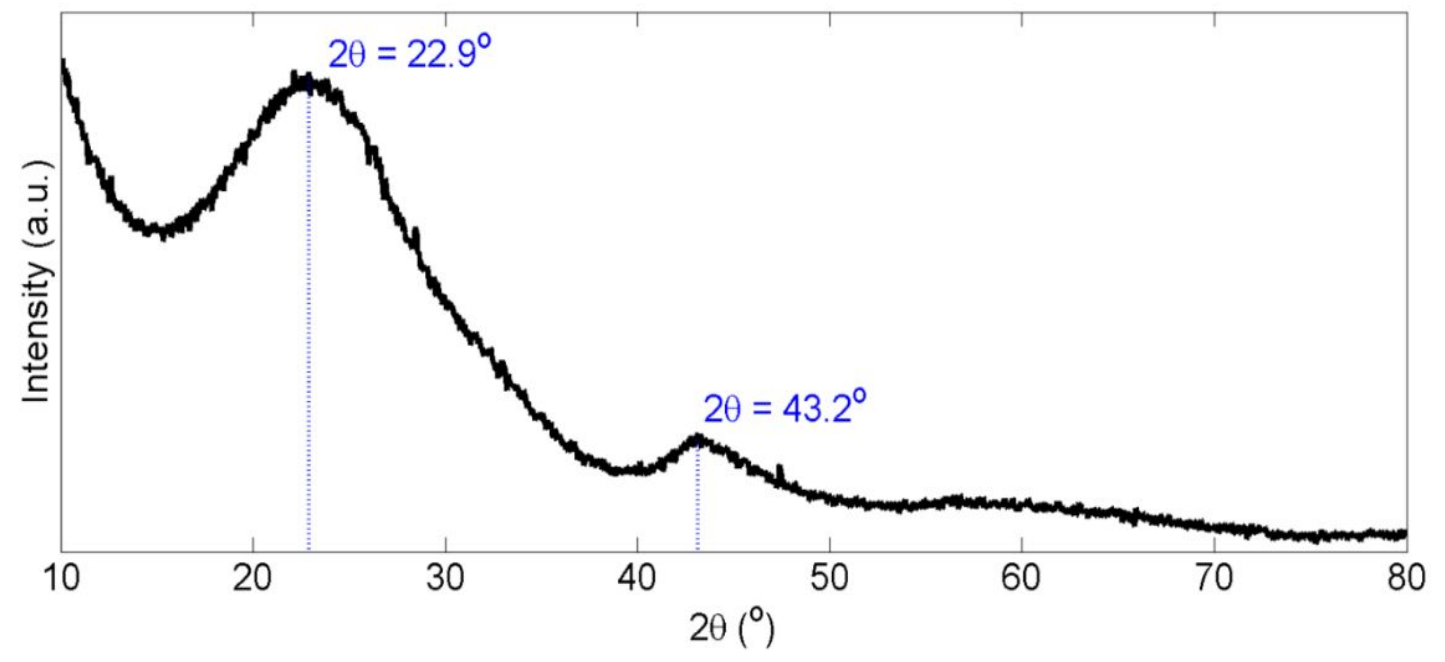

Figure S7 X-ray diffraction spectrum of LIG-fPI. The peak at $2 \theta=22.9^{\circ}$ and $43.2^{\circ}$ indicate (002) plane and (100) plane reflections in LIG-fPI, respectively. 


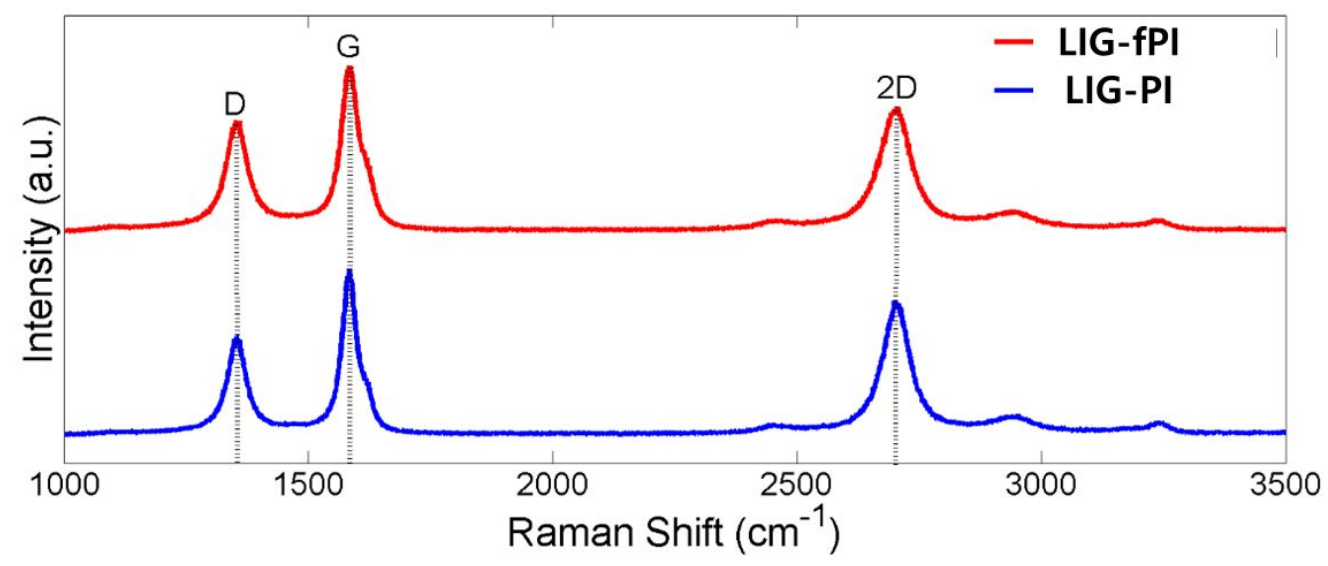

Figure S8 Raman spectra of LIG-fPI and LIG-PI. LIG-fPI and LIG-PI, fabricated with laser power of $1.8 \mathrm{~W}$ and $4.8 \mathrm{~W}$, respectively. 


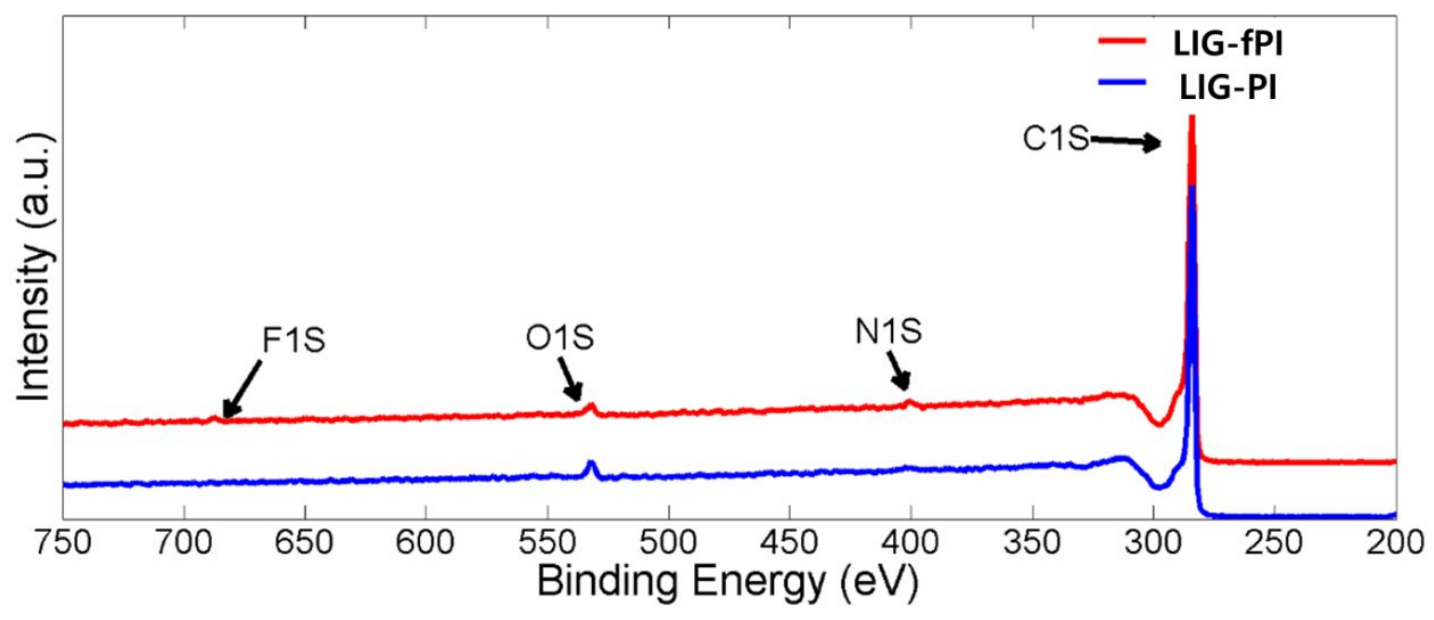

Figure S9 X-ray photoelectron spectra of LIG-fPI and LIG-PI. 


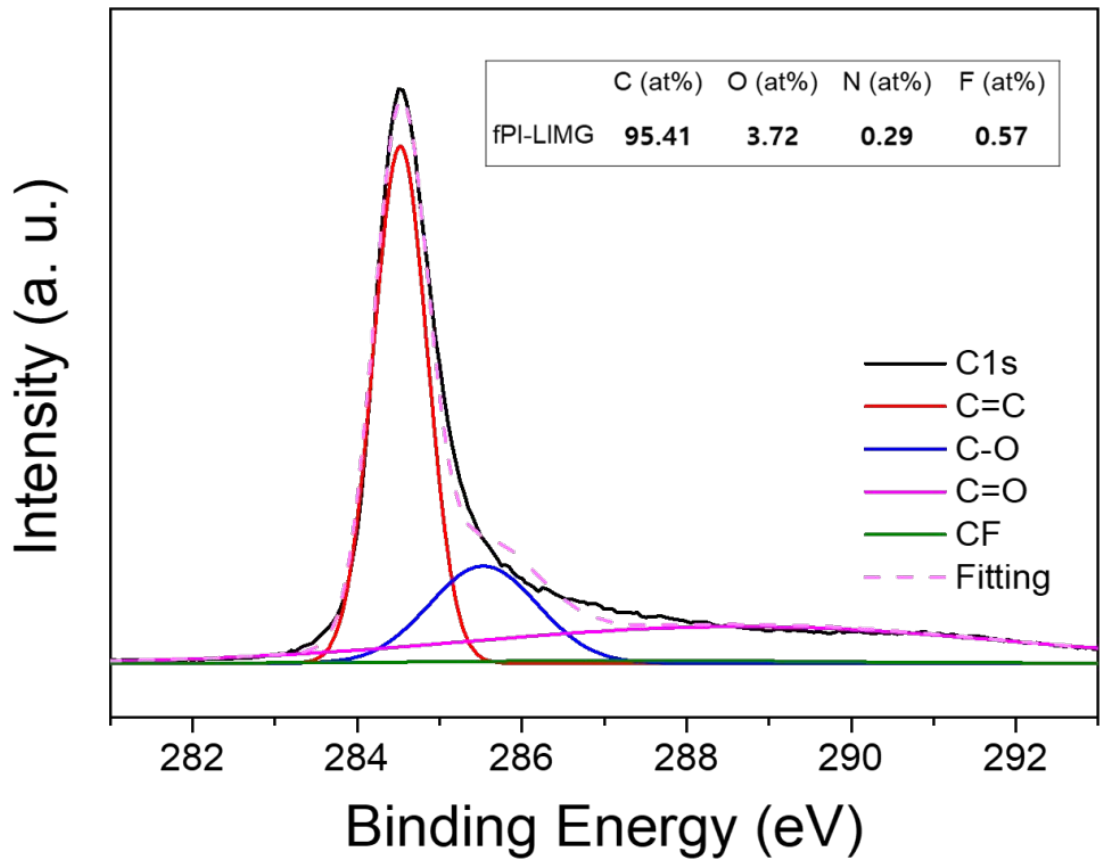

Figure S10 C1s XPS spectrum of LIG-fPI. 
a

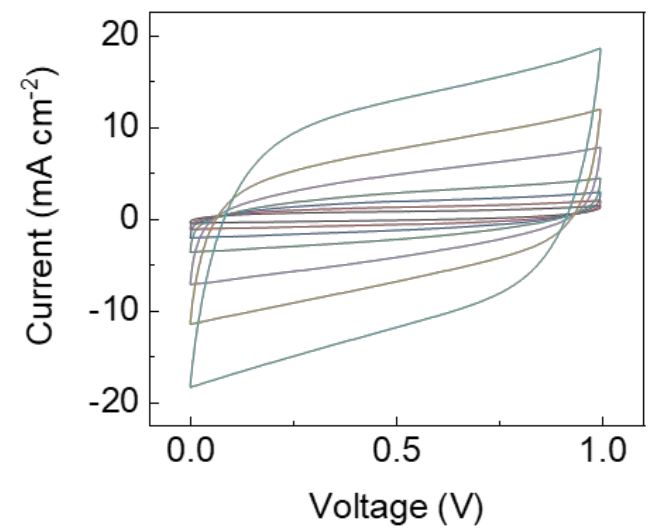

C

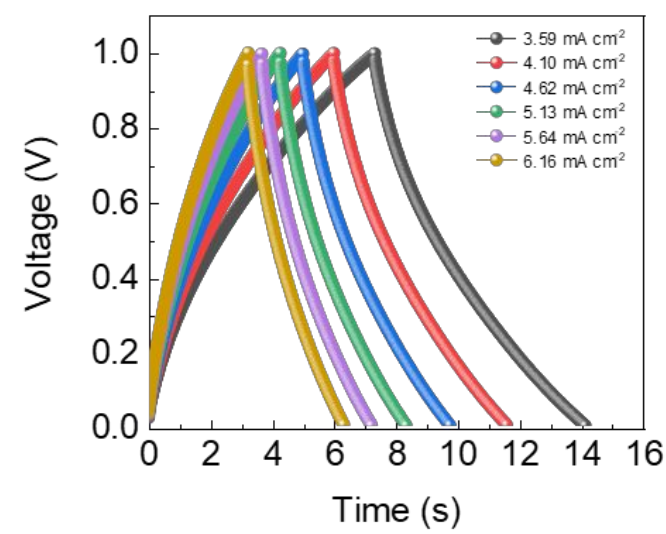

b

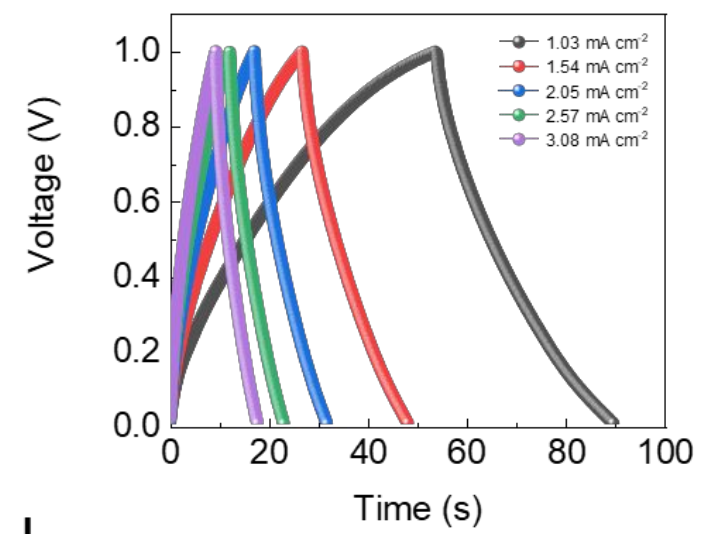

d

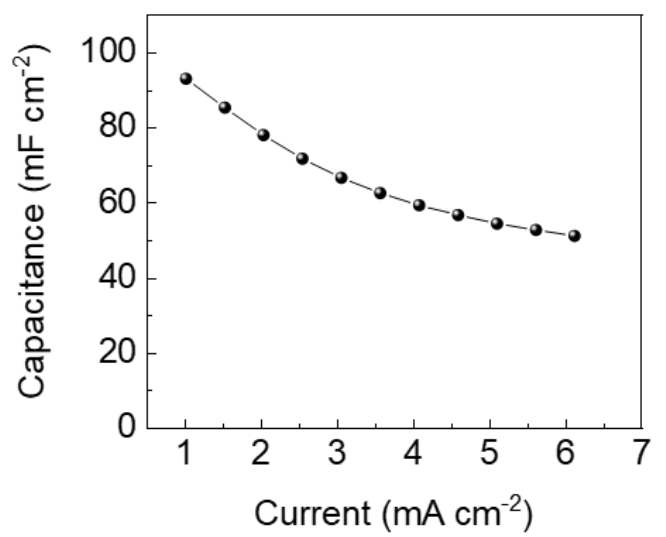

Figure S11 In $1 \mathrm{M} \mathrm{H}_{2} \mathrm{SO}_{4}$ aqueous solution, (a) CV curves (at scan rates ranging from 10 to $1000 \mathrm{mV}$ $\left.\mathrm{s}^{-1}\right),(\mathrm{b}, \mathrm{c}) \mathrm{GCD}$ profiles at different currents, and (d) rate-dependent areal capacitance of LIG-fPI-MSCs in a two-electrode system. 
a

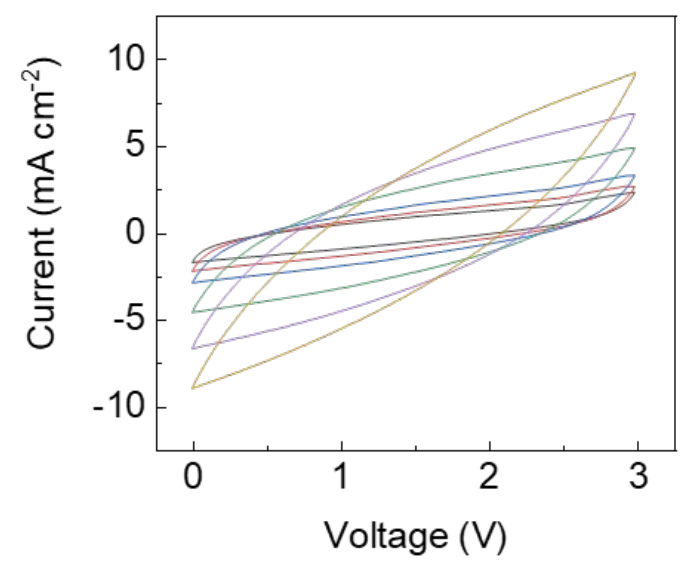

b

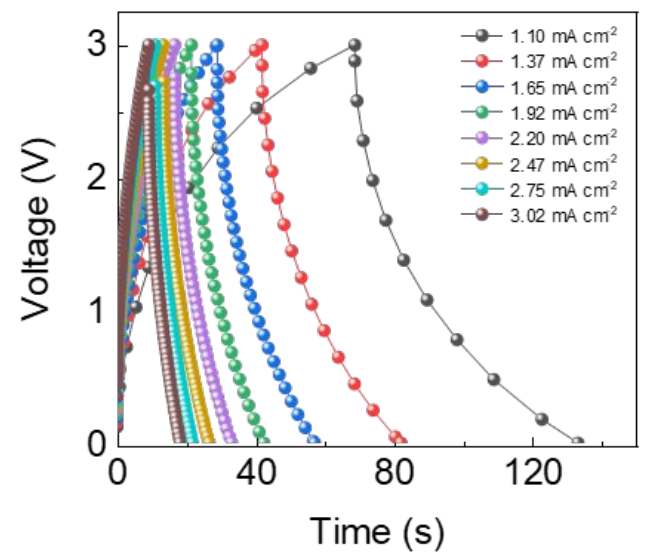

Figure S12 (a) CV curves and (b) GCD profiles of LIG-fPI-MSCs using o-GPE as a solid-state organic electrolyte. 


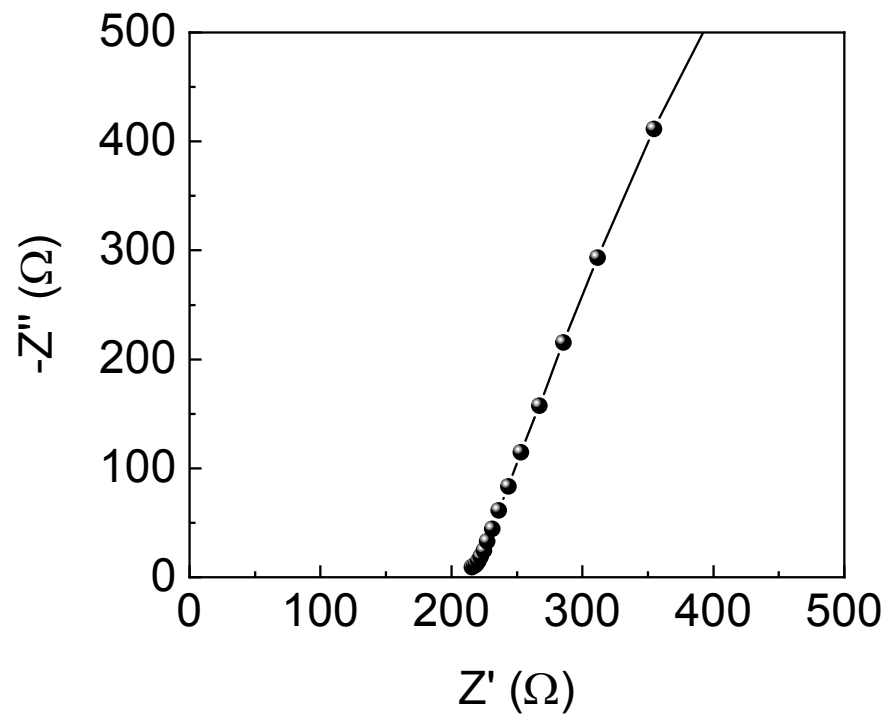

Figure S13 Nyquist plot of LIG-fPI-MSC employing o-GPE. 


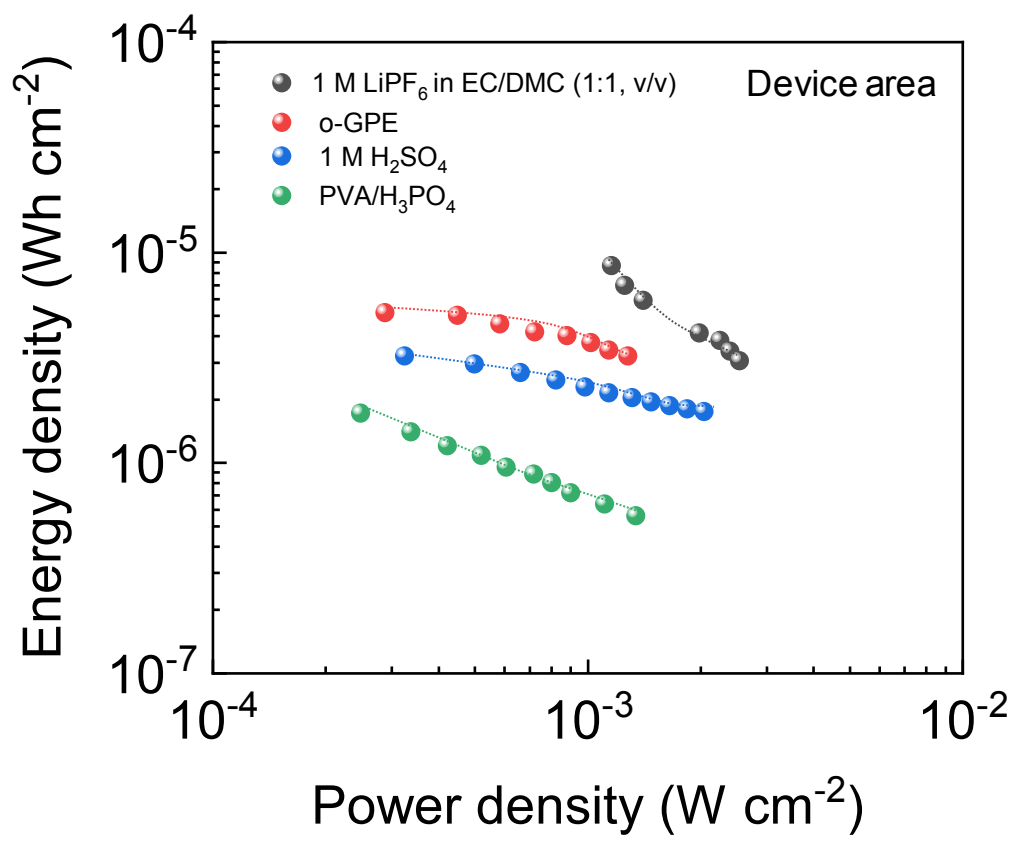

Figure S14 Device area-basis energy and power densities of LIG-fPI-MSCs with different electrolytes including $1 \mathrm{M} \mathrm{LiPF}_{6}$ in EC/DMC (1:1, v/v), o-GPE, $1 \mathrm{M} \mathrm{H}_{2} \mathrm{SO}_{4}$ aqueous electrolyte, and $\mathrm{PVA} / \mathrm{H}_{3} \mathrm{PO}_{4}$ solid-state electrolyte. 
a
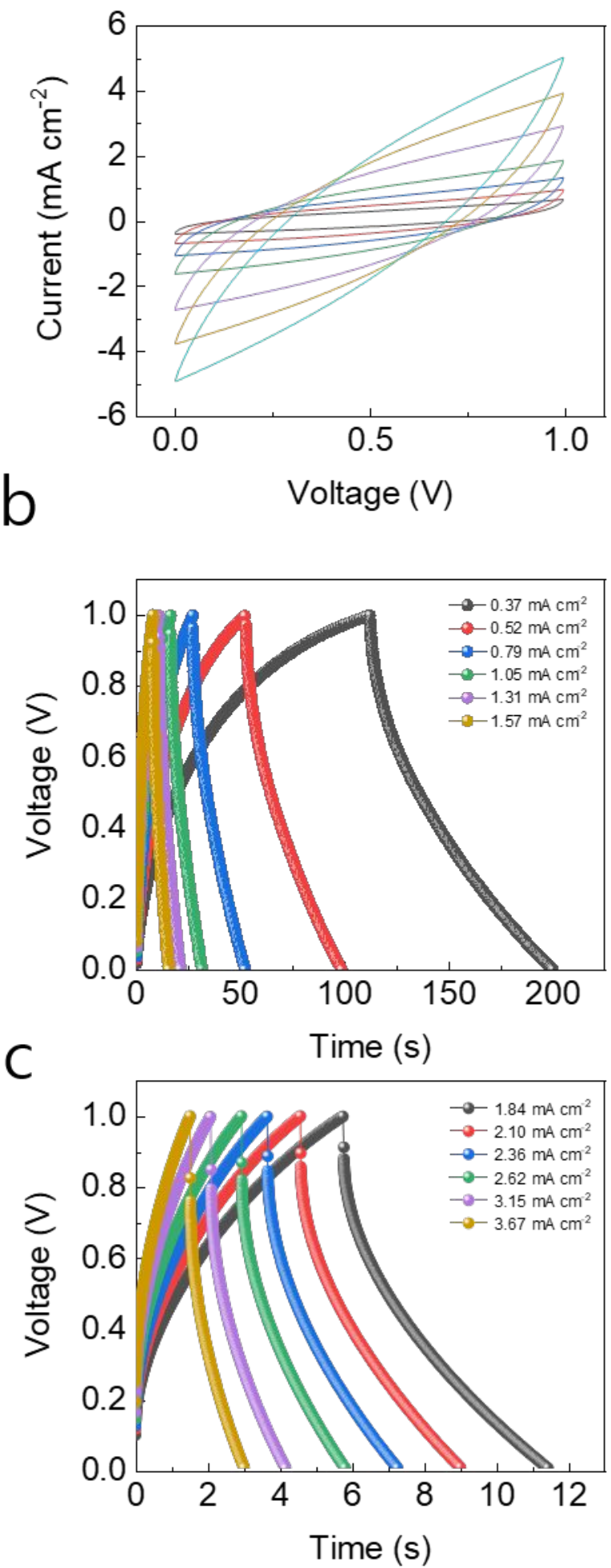

Figure S15 (a) CV curves (at scan rates ranging from 10 to $1000 \mathrm{mV} \mathrm{s}^{-1}$ ) and (b,c) GCD profiles at different currents of LIG-fPI-MSC using PVA/ $\mathrm{H}_{3} \mathrm{PO}_{4}$ solid-state electrolyte in a two-electrode. 


\section{Supporting Tables}

Table S1. The stabilization and imidization condition for the fluorinated polyimides films

\begin{tabular}{|c|c|}
\hline Step & Temperatures $\left({ }^{\circ} \mathrm{C}\right) /$ time $(\mathrm{h}) /$ oven \\
\hline Stabilization & $25 / 15 /$ vacuum $\rightarrow 50 / 1 /$ vacuum $\rightarrow 80 / 3 /$ vacuum \\
\hline \multirow{2}{*}{ Imidization } & $\begin{array}{c}110 / 0.5 / \mathrm{N}_{2} \rightarrow 130 / 0.5 / \mathrm{N}_{2} \rightarrow 160 / 0.5 / \mathrm{N}_{2} \rightarrow 190 / 0.5 / \mathrm{N}_{2} \\
\rightarrow 220 / 0.5 / \mathrm{N}_{2} \rightarrow 250 / 0.5 / \mathrm{N}_{2} \rightarrow 280 / 0.5 / \mathrm{N}_{2} \rightarrow 310 / 0.5 / \mathrm{N}_{2}\end{array}$ \\
\hline
\end{tabular}


Table S2. Comparison of areal capacitances of EDLC-type MSCs

\begin{tabular}{|c|c|c|c|c|}
\hline Electrodes & Electrolyte & $\begin{array}{c}\text { Voltage window } \\
\text { (V) }\end{array}$ & $\begin{array}{l}\text { Areal capacitance } \\
\qquad\left(\mathrm{mF} \mathrm{cm}^{-2}\right)\end{array}$ & Refs \\
\hline \multirow{2}{*}{ LIG-MSCs ${ }^{1)}$} & $1.0 \mathrm{M} \mathrm{H}_{2} \mathrm{SO}_{4}$ & 1 & 4.0 & \multirow{2}{*}[1]{} \\
\hline & $\mathrm{BMIM}-\mathrm{BF}_{4}$ & 3.5 & - & \\
\hline \multirow{2}{*}{ LSG-MSCs ${ }^{2)}$} & $\mathrm{PVA} / \mathrm{H}_{2} \mathrm{SO}_{4}$ & 1 & 2.3 & \multirow{2}{*}[2]{} \\
\hline & BMIM-NTf $_{2}$ & 2.5 & - & \\
\hline MPG-MSCs ${ }^{3)}$ & $\mathrm{PVA} / \mathrm{H}_{2} \mathrm{SO}_{4}$ & 1 & 0.32 & {$[3]$} \\
\hline \multirow{4}{*}{ LSG-ECs ${ }^{4)}$} & $\mathrm{PVA} / \mathrm{H}_{3} \mathrm{PO}_{4}$ & 1 & 3.7 & \multirow{4}{*}[4]{} \\
\hline & $1.0 \mathrm{M} \mathrm{H}_{2} \mathrm{SO}_{4}$ & 1 & 4.0 & \\
\hline & EMIM-BF $_{4}$ & 4 & 5.0 & \\
\hline & $1.0 \mathrm{M} \mathrm{TEA}^{-\mathrm{BF}_{4}}$ & 3 & 4.8 & \\
\hline \multirow{2}{*}{ RGO-GO-RGO } & $1.0 \mathrm{M} \mathrm{Na}_{2} \mathrm{SO}_{4}$ & 1 & 0.5 & \multirow{2}{*}[5]{} \\
\hline & $1.0 \mathrm{M}$ TEA-BF ${ }_{4}$ & - & 2.7 & \\
\hline rGO-CNT & $3.0 \mathrm{M} \mathrm{KCl}$ & 1 & 5.6 & {$[6]$} \\
\hline LIG-PI & $\mathrm{PVA} / \mathrm{H}_{3} \mathrm{PO}_{4}$ & 0.8 & 0.8 & {$[7]$} \\
\hline CNTs-based MSCs & $\mathrm{PVA} / \mathrm{H}_{3} \mathrm{PO}_{4}$ & 1 & 4.7 & {$[8]$} \\
\hline LIMDG $^{5)}$ & $\mathrm{PVA} / \mathrm{H}_{2} \mathrm{SO}_{4}$ & 0.8 & 1.4 & [9] \\
\hline B-LIG-MSCs $\left.{ }^{6}\right)$ & $\mathrm{PVA} / \mathrm{H}_{2} \mathrm{SO}_{4}$ & 1 & 16 & {$[10]$} \\
\hline \multirow{2}{*}{ LIG-MSCs } & $\mathrm{PVA} / \mathrm{H}_{2} \mathrm{SO}_{4}$ & 1 & 9.0 & \multirow{2}{*}[11]{} \\
\hline & $1.0 \mathrm{M} \mathrm{H}_{2} \mathrm{SO}_{4}$ & 1 & 2.0 & \\
\hline \multirow{2}{*}{ LIG-fPI-MSCs (This work) } & o-GPE & 3 & 16 & \multirow{2}{*}{ This work } \\
\hline & $1.0 \mathrm{M} \mathrm{H}_{2} \mathrm{SO}_{4}$ & 1 & 110 & \\
\hline
\end{tabular}

1) Laser-induced graphene microsupercapacitors

2) Laser-scribed graphene micro-supercapacitors

3) Reduced graphene based micro-supercapacitors

4) Laser-scribed graphene electrochemical capacitors

5) Laser-induced MOF-derived graphene

6) Boron-doped laser-induced graphene microsupercapacitors 
Table S3. Power and energy densities of EDLC-type MSCs

\begin{tabular}{|c|c|c|c|c|c|}
\hline Electrodes & Electrolyte & $\begin{array}{l}\text { Voltage window } \\
\text { (V) }\end{array}$ & $\begin{array}{l}\text { Power density } \\
\qquad\left(\mathrm{W} \mathrm{cm}^{-2}\right)\end{array}$ & $\begin{array}{l}\text { Energy density } \\
\qquad\left(\mathrm{Wh} \mathrm{cm}^{-2}\right)\end{array}$ & Refs \\
\hline \multirow{2}{*}{ LIG-MSCs } & $1.0 \mathrm{M} \mathrm{H}_{2} \mathrm{SO}_{4}$ & 1 & $9 \times 10^{-3}$ & $3 \times 10^{-7}$ & \multirow{2}{*}[1]{} \\
\hline & $\mathrm{BMIM}-\mathrm{BF}_{4}$ & 3.5 & $9 \times 10^{-3}$ & $2 \times 10^{-7}$ & \\
\hline \multirow{2}{*}{ LSG-MSCs } & $\mathrm{PVA} / \mathrm{H}_{2} \mathrm{SO}_{4}$ & 1 & $30 \times 10^{-3}$ & $2 \times 10^{-7}$ & \multirow{2}{*}[2]{} \\
\hline & BMIM-NTf 2 & 2.5 & $100 \times 10^{-3}$ & $1 \times 10^{-7}$ & \\
\hline B-LIG-MSCs & $\mathrm{PVA} / \mathrm{H}_{2} \mathrm{SO}_{4}$ & 1 & $1.2 \times 10^{-3}$ & $2 \times 10^{-6}$ & {$[10]$} \\
\hline LIG-MSCs & $\mathrm{PVA} / \mathrm{H}_{2} \mathrm{SO}_{4}$ & 1 & $9 \times 10^{-3}$ & $1 \times 10^{-6}$ & {$[11]$} \\
\hline \multirow{2}{*}{$\begin{array}{l}\text { LIG-fPI- } \\
\text { MSCs }\end{array}$} & o-GPE & 3 & $3 \times 10^{-3}$ & $1 \times 10^{-5}$ & \multirow{2}{*}{$\begin{array}{l}\text { This } \\
\text { work }\end{array}$} \\
\hline & $1.0 \mathrm{M} \mathrm{H}_{2} \mathrm{SO}_{4}$ & 1 & $4 \times 10^{-3}$ & $6 \times 10^{-6}$ & \\
\hline
\end{tabular}




\section{Supporting References}

[1] Lin, J.; Peng, Z.; Liu, Y.; Ruiz-Zepeda, F.; Ye, R.; Samuel, E. L. G.; Yacaman, M. J.; Yakobson, B. I.; Tour, J. M., Laser-induced porous graphene films from commercial polymers. Nat. Commun. 2014, $5(1), 5714$.

[2] El-Kady, M. F.; Kaner, R. B., Scalable fabrication of high-power graphene microsupercapacitors for flexible and on-chip energy storage. Nat. Commun. 2013, 4 (1), 1475.

[3] Wu, Z. S.; Parvez, K.; Feng, X.; Müllen, K., Graphene-based in-plane micro-supercapacitors with high power and energy densities. Nat. Commun. 2013, 4 (1), 2487.

[4] El-Kady, M. F.; Strong, V.; Dubin, S.; Kaner, R. B., Laser Scribing of High-Performance and Flexible Graphene-Based Electrochemical Capacitors. Science 2012, 335 (6074), 1326.

[5] Gao, W.; Singh, N.; Song, L.; Liu, Z.; Reddy, A. L. M.; Ci, L.; Vajtai, R.; Zhang, Q.; Wei, B.; Ajayan, P. M., Direct laser writing of micro-supercapacitors on hydrated graphite oxide films. Nat. Nanotechnol. 2011, 6 (8), 496-500.

[6] Beidaghi, M.; Wang, C., Micro-Supercapacitors Based on Interdigital Electrodes of Reduced Graphene Oxide and Carbon Nanotube Composites with Ultrahigh Power Handling Performance. $A d v$. Funct. Mater. 2012, 22 (21), 4501-4510.

[7] In, J. B.; Hsia, B.; Yoo, J.-H.; Hyun, S.; Carraro, C.; Maboudian, R.; Grigoropoulos, C. P., Facile fabrication of flexible all solid-state micro-supercapacitor by direct laser writing of porous carbon in polyimide. Carbon 2015, 83, 144-151.

[8] Yu, W.; Zhou, H.; Li, B. Q.; Ding, S., 3D Printing of Carbon Nanotubes-Based Microsupercapacitors. ACS Appl. Mater. Interfaces 2017, 9 (5), 4597-4604.

[9] Basu, A.; Roy, K.; Sharma, N.; Nandi, S.; Vaidhyanathan, R.; Rane, S.; Rode, C.; Ogale, S., CO2 Laser Direct Written MOF-Based Metal-Decorated and Heteroatom-Doped Porous Graphene for Flexible All-Solid-State Microsupercapacitor with Extremely High Cycling Stability. ACS Appl. Mater. Interfaces 2016, 8 (46), 31841-31848.

[10] Peng, Z.; Ye, R.; Mann, J. A.; Zakhidov, D.; Li, Y.; Smalley, P. R.; Lin, J.; Tour, J. M., Flexible Boron-Doped Laser-Induced Graphene Microsupercapacitors. ACS Nano 2015, 9 (6), 5868-5875.

[11] Peng, Z.; Lin, J.; Ye, R.; Samuel, E. L. G.; Tour, J. M., Flexible and Stackable Laser-Induced Graphene Supercapacitors. ACS Appl. Mater. Interfaces 2015, 7 (5), 3414-3419. 